\title{
Complex lung function in severe asthma: seeing is believing
}

\author{
Gregory G. King ${ }^{1,2,3}$ and Cindy Thamrin ${ }^{1}$
}

Affiliations: 'Woolcock Institute of Medical Research, The University of Sydney, Australia. ${ }^{2}$ Dept of Respiratory Medicine, Royal North Shore Hospital, St Leonards, Australia. ${ }^{3}$ NHMRC Centre of Excellence in Severe Asthma.

Correspondence: Gregory G. King, Royal North Shore Hospital - Respiratory Medicine, Pacific Highway, St Leonards, New South Wales 2065, Australia. E-mail: gregory.kinglasydney.edu.au

@ERSpublications

Techniques to partition airway dysfunction could improve understanding of severe asthma http://ow.ly/N7zD301wFn6

Difficult-to-treat or severe asthma remains a common problem and a challenge for healthcare providers. It affects up to $10 \%$ of asthmatic individuals, who continue to suffer high disease burden and healthcare costs [1]. This clinical problem still exists despite many advances in the understanding of the underlying basis of asthma and improved management strategies, which include targeted anti-inflammatory therapy with monoclonal antibodies. Research effort continues, with exploration of different aspects of pathophysiology such as genomics, proteomics, inflammation, airway remodelling and airway smooth muscle abnormalities. In this context, the examination of complex lung function at the whole-organ level becomes increasingly important. There have been many technical advances in functional imaging and in complex measures of lung function that can now be used to explore the pathophysiology of asthma at the whole-organ level, and which can be more directly related to clinical manifestations of disease such as symptoms and airway hyperresponsiveness.

Measuring patterns of ventilation (ventilation distribution) is a way to characterise lung function that is highly relevant in obstructive lung diseases, and goes far beyond spirometry, peak flow and lung volumes. Ventilation distribution can be quantified visually using three-dimensional (tomographic) ventilation imaging techniques as well as functionally using inert-gas washout tests. Tomographic imaging methods include hyperpolarised noble gas magnetic resonance imaging (MRI) (e.g. helium-3 or xenon) [2], positron emission tomography [3], single photon emission computed tomography (CT) $[4,5]$ and oxygen enhanced MRI [6], where the distribution of a tracer gas is imaged. Inert-gas washout tests are based on the simple principle that regions of the lung that ventilate poorly or not at all (i.e. altered distribution) delay the clearance (i.e. washout) of a marker gas from the lung during normal tidal breathing. Thus, $100 \%$ oxygen can be used to "wash out" the resident nitrogen over multiple breaths, which is the basis of the multiple-breath nitrogen washout (MBNW). Similarly, if low concentrations of inert gases such as sulfur hexafluoride or helium are introduced into the lung, then their pattern of clearance from the lung reflects ventilation distribution.

Ventilation imaging and inert-gas washout tests are thus two different yet complementary methods for characterising ventilation distribution. Ventilation imaging shows where functional abnormalities occur, how extensive they are and how they are colocated. Conversely, inert-gas washout measures ventilation that has been averaged across the entire lung but probes much finer length scales than those afforded by the resolution of modern imaging techniques. Ventilation distribution measured from inert-gas washouts is determined by the small airways; studies in smokers [7] and bone marrow transplant recipients [8] suggest it to be a highly sensitive test of small-airway function. Furthermore, analysis of the washout pattern over the course of the washout has allowed compartmentalisation of function into acinar and conductive small airways, which has also been shown to have separate clinical associations in airway

Received: June 052016 | Accepted: June 132016

Conflict of interest: Disclosures can be found alongside this article at erj.ersjournals.com

Copyright (CERS 2016 
diseases [9-12]. The lung clearance index (LCI) is a simpler and "aggregate" measure of airway function, and represents the number of breaths, adjusted for lung size, required to clear the lungs of a gas.

The body of evidence from inert-gas washout studies in asthma shows that there is increased heterogeneity of ventilation, i.e. ventilation is more variable within the diseased lung. This means that there are areas of the lung that are poorly ventilated, which can be present either at baseline or during induced bronchoconstriction, that is, the inflammation and tissue remodelling of airways and of the lung in asthma lead to functional impairment in only some airways (ventilation heterogeneity) rather than being uniformly distributed across the whole lung. So far, this is perhaps not surprising. The significance, though, is that ventilation heterogeneity has strong associations with airway inflammation measured by exhaled nitric oxide [9], airway hyperresponsiveness [9, 12], asthma symptoms and control [10, 11], and it is associated with moderate or greater exacerbations [13]. Furthermore, it improves with inhaled corticosteroid treatment and is accompanied by improvements in asthma control [11]. Ventilation distribution, therefore, is likely to be highly relevant to the better understanding of severe asthma and, in particular, understanding why there may be an incomplete response to inhaled corticosteroid and long-acting bronchodilator treatment.

The main contribution of imaging studies to our understanding of ventilation distribution in asthma is the revelation of the presence of large and dramatic ventilation defects $[3-5,14]$, caused presumably by both localised airway closure and severe airway narrowing. There has been very little analysis of the distribution of ventilation, i.e. reduced ventilation due to airway narrowing per se [15]. Perhaps this because researchers' attentions have been drawn to the visually dramatic nature of ventilation defects, which again have been elegantly demonstrated using hyperpolarised ${ }^{3} \mathrm{He}$ MRI by SvENNINGSEN et al. [2] in this issue of the European Respiratory Journal. It is uncertain what causes these defects but the fact that they tend to persist over time [16] and can be induced acutely by bronchoconstriction [3, 4, 14], which is also repeatable between sessions [14], suggests a structural basis to their presence, rather than being due simply to mucus plugging. Computational modelling studies suggest that these ventilation defects arise from widespread small-airway narrowing combined with severe narrowing in some of the larger airways [17]. Heterogeneous airway narrowing in medium-to-large airways has been confirmed in CT studies of airway narrowing $[18,19]$ but this has never been related to ventilation imaging. This serves to remind us that in asthma, and possibly more so in severe asthma, both small and large airways are likely play important roles in producing recurrent airway narrowing and closure that may at times be catastrophic.

So what does all this mean, particularly in the context of severe asthma? Firstly, patchy ventilation in small airways as measured by gas washout studies is important functionally, given its relationship to airway hyperresponsiveness, as well as clinically, because of its relationship with asthma symptoms and control. Secondly, there are patches of the lung and airways that are badly affected and are highly dysfunctional. When bronchoconstricted, these defects increase further; it is presumed the baseline ventilation defects indicate lung regions that are the worst affected while the regions that closed when bronchoconstricted are hyperresponsive and hyperreactive. If these lung regions were mechanistically responsible for asthma symptoms, then one could postulate that the key to treating difficult-to-control asthma lies within these areas. Hence, this lends significance to the associations between ventilation defects and asthma control reported by SVENNINGSEN et al. [2], given that they studied patients with quite severe asthma who were relatively young (mean age 46 years) who were highly symptomatic, with high Asthma Control Questionnaire (ACQ) scores, had a range of airflow obstruction (forced expiratory volume in $1 \mathrm{~s} /$ forced vital capacity ratio down to 0.33 ) and nearly half were oral corticosteroid dependent.

An important question raised by the observations of SVENNINGSEN et al. [2] is whether ventilation defects could directly contribute to asthma symptoms? The ACQ is based on retrospective recall of symptoms from the previous week, which could conceivably be attributed to spontaneous airway narrowing and closure. In this study, ventilation defects related strongly to ACQ. So why should ventilation defects at one point in time relate to intermittent airway narrowing and closure in the recent past? One reason could be, as suggested by the authors, that ventilation defects are an indicator of reactive and hyperresponsive airways elsewhere that will close when stimulated, as has been previously demonstrated, rather than being directly responsible in their own right for asthma severity.

It is clear that longitudinal treatment studies are required in severe asthma with ventilation imaging as one potential outcome, in order to see if targeting a lung region could be beneficial. However, there are other remaining questions. What is blocking ventilation when the patient is clinically stable? If it is inflammation, why is it not responsive to high-dose corticosteroid treatment? If it is due to tissue remodelling, this would explain a lack of corticosteroid responsiveness but how could this be treated? MBNW indices specific to acinar and conductive airway function may be more sensitive to regional ventilation defects than the LCI used in the SvENNINGSEn et al. [2], and should ideally be used in future studies. Are there more practical ways than ${ }^{3} \mathrm{He}$ MRI to determine regional lung response to experimental treatments of severe asthma? Although 
sophisticated and expensive ventilation imaging can be justified in proof-of-concept studies, more practical methods will be needed in later stage studies, for example, washout tests and oscillatory impedance techniques could be considered. Is it possible to target these poorly ventilated and closed lung regions with aerosol therapy, given airflow is required to deliver the therapeutic aerosols? Finally, what is the significance of airway narrowing, i.e. poorly ventilated lung regions (as distinct from ventilation defects), which potentially indicate unstable lung regions that need attention?

In summary, the findings of SvenNingsen et al. [2] demonstrate the clinical importance of regional lung function in severe asthma and offer a potential mechanism particularly for non-steroid responsive asthma. Further characterisation of the lungs in severe asthma, either using functional imaging or indices from complex lung function that enable the partitioning of airway dysfunction, would add to our understanding and improve our ability to identify relevant regions for targeting therapy.

\section{References}

1 Busse WW. Difficult-to-treat asthma: how serious is the problem and what are the issues? In: Chung KF, Bel EH, Wenzel SE, eds. Difficult-to-Treat Severe Asthma (ERS Monograph). Sheffield, European Respiratory Society, 2011; pp. 1-15.

2 Svenningsen S, Nair P, Guo F, et al. Is ventilation heterogeneity related to asthma control? Eur Respir J 2016; 48: 370-379.

3 Harris RS, Winkler T, Tgavalekos N, et al. Regional pulmonary perfusion, inflation, and ventilation defects in bronchoconstricted patients with asthma. Am J Respir Crit Care Med 2006; 174: 245-253.

4 Farrow CE, Salome CM, Harris BE, et al. Airway closure on imaging relates to airway hyperresponsiveness and peripheral airway disease in asthma. J Appl Physiol 2012; 113: 958-966.

5 King GG, Eberl S, Salome CM, et al. Differences in airway closure between normal and asthmatic subjects measured with single-photon emission computed tomography and technegas. Am J Respir Crit Care Med 1998; 158: 1900-1908.

6 Sá RC, Cronin MV, Cortney Henderson A, et al. Vertical distribution of specific ventilation in normal supine humans measured by oxygen-enhanced proton MRI. J Appl Physiol 2010; 109: 1950-1959.

7 Verbanck S, Schuermans D, Meysman M, et al. Noninvasive assessment of airway alterations in smokers: the small airways revisited. Am J Respir Crit Care Med 2004; 170: 414-419.

8 Lahzami S, Schoeffel R, Pechey V, et al. Small airways function declines after allogeneic hematopoietic stem cell transplantation. Eur Respir J 2011; 38: 1180-1188.

9 Downie S, Salome C, Verbanck S, et al. Ventilation heterogeneity is a major determinant of airway hyperresponsiveness in asthma, independent of airway inflammation. Thorax 2007; 62: 684-689.

10 Farah CS, King GG, Brown NJ, et al. The role of the small airways in the clinical expression of asthma in adults. J Allergy Clin Immunol 2012; 129: 381-387.

11 Farah CS, King GG, Brown NJ, et al. Ventilation heterogeneity predicts asthma control in adults following inhaled corticosteroid dose titration. J Allergy Clin Immunol 2012; 130: 61-68.

12 Hardaker KM, Downie SR, Kermode JA, et al. The predictors of airway hyperresponsiveness differ between old and young asthmatics. Chest 2011; 139: 1395-1401.

13 Bourdin A, Paganin F, Préfaut C, et al. Nitrogen washout slope in poorly controlled asthma. Allergy 2006; 61: 85-89.

14 de Lange EE, Altes TA, Patrie JT, et al. The variability of regional airflow obstruction within the lungs of patients with asthma: assessment with hyperpolarized helium-3 magnetic resonance imaging. J Allergy Clin Immunol 2007; 119: 1072-1078.

15 Tzeng Y-S, Lutchen $\mathrm{K}$, et al. The difference in ventilation heterogeneity between asthmatic and healthy subjects quantified using hyperpolarized ${ }^{3} \mathrm{He}$ MRI. J Appl Physiol 2009; 106: 813-822.

16 de Lange EE, Altes TA, Patrie JT, et al. Changes in regional airflow obstruction over time in the lungs of patients with asthma: evaluation with ${ }^{3} \mathrm{He}$ MR imaging. Radiology 2009; 250: 567-575.

17 Campana L, Kenyon J, Zhalehdoust-Sani S, et al. Probing airway conditions governing ventilation defects in asthma via hyperpolarized MRI image functional modeling. J Appl Physiol 2009; 106: 1293-1300.

18 Dame Carroll J, Magnussen J, Berend N, et al. Greater parallel heterogeneity of airway narrowing and airway closure in asthma measured by high resolution computed tomography. Thorax 2015; 70: 1163-1170.

19 King GG, Carroll JD, Müller NL, et al. Heterogeneity of narrowing in normal and asthmatic airways measured by HRCT. Eur Respir J 2004; 24: 211-218. 